\title{
Analyse transversale des activités et paradigme de la transformation
}

\begin{abstract}
Abstrait
Le but de cet article, intentionnellement préparé pour le numéro thématique "Pensée transformation » est de montrer comment l'analyse transversale des activités et le paradigme de la transformation, présents dans l'œuvre de Jean-Marie Barbier $(2016,2017)$, traversent également la perspective socio-pédagogique.

Dans le texte, nous réfléchissons sur les cadres épistémologiques et les caractéristiques de l'approche transversale ainsi que sur ses relations avec le paradigme de la transformation. Le concept d'analyse transversale de l'activité et ses liens avec le paradigme transversal de la transformation en pédagogie sociale sont également introduits.
\end{abstract}

Mots-clés : transversale analyse des activités, paradigme de la transformation, pédagogie sociale, raison transversale.

\section{Transversal Activity Analysis and the Transformation Paradigm}

\begin{abstract}
The aim of this article, intentionally prepared for the thematic issue "Thinking transformations", is to discuss how the transversal approach to the analysis of activity and the paradigm of transformation present in Jean-Marie Barbier's work permeate the socio-pedagogical perspective. The text discusses the epistemological framework and features of the transversal approach as well as its relationship to the transformation paradigm. The concept of the transversal analysis of activity and its connections with the transversal paradigm of transformation in social pedagogy is also introduced.
\end{abstract}

Keywords: transversal activity analysis, transformation paradigm, social pedagogy, transversal reason.

\footnotetext{
* Université de Lodz, Pologne.

Article soumis le 3 février 2021 ; accepté pour publication le 4 mars 2021.
} 


\section{Point de départ}

Le but de cet article, intentionnellement préparé pour le numéro thématique "Pensée transformation » est de montrer comment le paradigme de la transformation, présent dans l'œuvre de Jean-Marie Barbier $(2016,2017)$, traverse également la perspective socio-pédagogique d'analyse transversale des activités.

Entrer dans le milieu de chercheurs partageant des idées et outils de pensée communs est habituellement une expérience significative pour transformer la posture du chercheur. Pour nous, les résultats de ce processus consistent à assimiler les concepts présents dans la nouvelle proposition d'intelligibilité de l'activité dans le champ de pratiques. Le noyau le plus important de cette perspective est une nouvelle façon de penser sur le mode de transformation des processus se déroulant dans différents espaces : éducatif, culturel, personnel, social, de prévention, etc.

Cet article est un exemple de transformation multidimensionnelle de point de vue sur les problématiques de l'activité et du sujet agissant, et, par conséquent, de la perception transversale de l'activité dans un champ de pratique. À la suite d'une recherche conjointe et d'une inter-pénétration des pensées et positions scientifiques rencontrées, il a été possible de construire un nouveau paradigme de pédagogie sociale. Dans ses fondements, nous retrouvons bien les liens avec l'épistémologie de l'équipe dirigée par Jean-Marie Barbier.

\section{Transversalité : cadres d'analyse}

Une posture de pensée se construit en traversant les expériences partagées des participants à l'activité. Ces idées imprègnent transversalement les représentations sociales des sujets, et construisent une institution symbolique. Elle est de nature relationnelle et modifie les activités mentales du sujet.

La caractéristique de ces activités est leur transversalité. La compréhension des mécanismes transversaux des activités mentales devient alors un défi. L'un des concepts expliquant ce processus est la proposition de Wolfgang Welsch (1998b : 98), connue sous le nom de : "raison transversale ».

Une catégorie particulièrement importante dans ce concept est la « transition » où les vieilles idées/représentations en rencontrent de nouvelles et se « déplacent » vers une nouvelle forme.

L'esprit transversal est libre de « structurer le monde en ensembles spécifiques, et donc il passe librement d'une rationalité à une autre. Il crée des liens, conduit à des tensions et à des transformations » (Kubicki 1998 : 165). Le concept d'esprit transversal décrit « le mode optimal de participation des individus à diverses pratiques culturelles de postmodernité. Chaque domaine de la culture, et même chaque activité, peut avoir sa propre rationalité » (ibidem : 165). 
Le traits caractéristiques de l'esprit transversal sont des tensions et des transformations. En effet, il produit des «tensions » et vit grâce à eux. Son image est hybride. Les mécanismes des transformations sont bien décrits par le concept de la transculturalité de Welsch (1998a). La " capacité de connexion et de transformation » est ici dominante (ibidem : 213). Elle permet de découvrir ce qui est « universel et commun » et de s'orienter vers une approche globale et holistique de l'activité. Dans le processus de transculturalité, les ressources/l'héritage, « les réservoirs " (ibidem : 210) sont importants. Ils constituent des points de vue (et des références) spécifiques à partir desquels d'autres «réseaux» sont créés. Ce processus de transculturalité déclenche une dimension transversale des références existantes, " des réservoirs ». Ce mécanisme de construction de la dimension transversale permet également de transgresser « des modèles/exigences/schèmes de comportement (par exemple habitus) - ou des points de vue monoculturels, donnés extérieurement » (ibidem : 210).

Cependant, les idées/représentations existantes, "les réservoirs », sont une ressource indispensable pour cultiver la perspective transversale. Elles se transforment en de nombreuses modifications hybrides, en liens conjoints (Barbier 2016, 2017). De cette manière, elles se modifient elles-mêmes, en perdant pour la dimension transversale la construction structurelle (horizontale/verticale). Cette dimension transversale infiltre les points de vue existants et les nouvelles ouvertures embrassant « des processus d'unification et de différenciation » (Welsch 1998a : 221). Ces processus sont brièvement exprimés par l'expression unité dans la diversité (Marynowicz-Hetka 2008).

Pourtant cette expression ne doit pas être comprise de façon équivoque. De même que dans le concept de la transculturalité, il ne s'agit pas de "simple uniformisation » (Welsch 1998a : 217) au cours de la transformation. Par effet d'infiltration dans ce processus, la diversité antérieure établit un nouveau type de

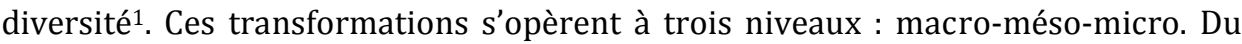
point de vue socio-éducatif, aucun d'entre eux ne peut être ignoré. Les niveaux macro et méso ne sont pas seulement, comme nous le pensons intuitivement, la conditionnalité sociale des phénomènes sociaux, mais surtout ce que Radlińska appelait le sol (Radlińska 1935 ; Witkowski 2014), et ce que maintenant Welsch (1998a : 204) appelle « la construction des réseaux » au travers et par lesquels un nouveau point de vue transversal se constitue. Le niveau micro exprime la « formation transculturelle des individus qui deviennent des hybrides culturels » (ibidem : 207).

\footnotetext{
1 Ce mécanisme de constitution d'une unité dans la diversité est cohérent avec le mécanisme de transformation transculturelle discuté par Welsch (1998a). L'auteur écrit ainsi : «La diversité traditionnelle, contenue ou forme de cultures individuelles, est en effet en train de disparaître de plus en plus. Mais à sa place, un nouveau type de diversité émerge : les cutures et les formes de vie différentes, dont chacune résulte d'une interpénétration transculturelle et a un style transculturel » (ibidem : 217).
} 
Ceci, semble très inquiétant. Comment éviter la relativisation de l'activité entreprise, son jugement, son orientation ? Quelle est l'importance de cette question?

L'idée de la perception transversale du réel est visible dans l'œuvre de Radlińska, ouverte à l'altérité/au changement/à la nouveauté, mais aussi protégeant le passé et s'y référant dans la construction du présent. Dans ce raisonnement, la catégorie de la génération historique occupe une place particulière. Elle détruit les frontières démographiques, culturelles et sociales, infiltre l'activité de tous ceux qui participent à la vie sociale dans une période historique donnée. La connaissance des mécanismes de constitution de la génération historique est également importante pour découvrir la qualité des relations sociales dans une société diversifiée, par principe. Créer une communauté partageant des préférences de valeur, nécessite de la part des participants une prise de conscience des liens transversaux qui la transforment en une génération historique dans laquelle se construit le sentiment d'expérience partagée. Ce phénomène porte le nom de filiation.

La transversalité entendue comme création d'une perspective transversale est particulièrement applicable dans le processus de disciplinarisation de la réflexion sur l'activité dans le champ de pratiques (cf. : Foucart 2016). Elle devient une réflexion sur la réflexion dans le processus de création des disciplines multiréférencielles, comme p.ex. sciences (ou science) de l'éducation ou science (ou des sciences) du travail social. En ce sens, l'approche transversale constitue une dimension de réflexion métathéorique sur les champs de pratiques éducatives ou sociales. Dans ce processus, la perspective transversale est visible. Notamment dans la construction d'une métathéorie embrassant des références épistémologiques pour l'intelligibilité de l'activité ${ }^{2}$, de la transformation, et d'autres concepts utiles pour comprendre les champs d'action professionnelle et les raisons d'orientation de l'activité.

Quand l'approche transversale (cf. Barbier 2006, 2016, 2017) est utilisée pour l'analyse des activités, elle prend en compte dans la recherche la perspective " sur» le champ de pratiques. Ceci permet une analyse complète de l'activité à distance en trois dimensions : « à l'extérieur », " à l'intérieur » et « au-dessus du champ d'activité ». Cette perspective permet une compréhension globale et complémentaire de l'activité dans les champs de pratiques. Elle fait prendre de l'importance à l'activité entreprise, permet l'analyse d'une situation donnée, en tenant compte de l'impact réciproque des trois catégories de représentations du sujet agissant : la situation dans laquelle l'activité est entreprise, le processus d'action et soi-même en tant que sujet agissant. Ces trois catégories de

\footnotetext{
2 Discutant de l'entrée activité, Jean-Marie Barbier et Marc Durand parlent d'une ou de plusieurs cultures « de pensée transversale (s) à plusieurs disciplines et champs de pratiques » (Barbier, Durand $2003: 104)$.
} 
représentations évoluent et se transforment au cours du processus de résolution du problème.

Du point de vue socio-pédagogique, une signification particulière est attribuée au marquage axiologique et symbolique ainsi qu'à la valorisation des liens entre les représentations : de ce qui existe et ce qui est désiré/attendu. Cette proposition (Barbier, Galatanu 2006) s'appuie sur la supposition que la perception de l'activité transversale nécessite une analyse de trois perspectives axiologiquement définies simultanément : mentale, affective et d'action.

Ainsi la perspective mentale contient une représentation de l'activité planifiée, de soi-même, de ses capacités et ses potentiels. La perspective affective comprend l'estime de soi en tant que sujet entreprenant une activité, ses compétences et le niveau d'agentivité. La perspective opérationnelle fait référence au déroulement du processus de l'activité/de l'action. Il est également important d'analyser le langage, en notant : ce qui est dit sur l'activité dans le domaine de la pratique, ce à quoi on prête attention, ce qui passe sous silence/n'est pas mentionné.

Pour conclure cette analyse : le concept d'esprit transversal unit la compréhension et la critique (Welsch 1998b : 98). Cela est possible parce que « chaque point de vue éclaire aussi d'autres perspectives et découvre des prémisses dont nous ne sommes pas conscients » (ibidem). Une telle compréhension attentive nous conduisait en général ipso facto à la critique et au changement de paradigme, lorsque l'échelle des changements est complètement remise en question » (ibidem). Pour cette raison, ce concept semble très attractif pour approfondir le paradigme de 'pensée transformation' (Barbier 2020 ; Barbier, Dutoit 2020).

\section{Longue durée, rupture et discontinuité}

Plusieurs positions peuvent constituer le cadre méthodologique du processus de transformation des activités mentales.

La première est la proposition de la catégorie conceptuelle de « longue durée » (Braudel 1999 [1971], 1949). L'auteur s'inquiétant de la communauté des sciences humaines, qui est si spécialisée sur son domaine et différenciée, appelle à une analyse globale et complète des phénomènes sociaux. Cette analyse nécessite, tout d'abord, de se souvenir de la nature cyclique des événements et de leur structure interne. Ce qui facilite aussi d'entreprendre cette position épistémologique, c'est le regard d'une autre perspective ou discipline, une certaine forme d'éloignement, de prise de distance. L'analyse des événements dans une perspective d'éloignement et de longue durée permet leur valorisation autonome. Cette perspective de longue durée donne lieu également à découvrir les sources de création de soi-même et de construction d'institution symbolique, grâce auxquelles elle confirme la conviction que les retours vers le passé sont utiles. Bien sûr, ils ne sont utiles que dans la mesure où ils nous facilitent l'explication du présent et fournissent des arguments 
et du matériel pour le projet futur. Ce n'est que dans la longue durée des générations historiques qu'il est possible de distinguer des éléments permanents d'une certaine approche, d'une position. La proposition de Braudel s'inscrit très bien dans l'approche transversale du paradigme de la pensée de transformation.

Le deuxième élément du cadre méthodologique du processus transversal de transformation des activités mentales est le concept de Bachelard des ruptures et des discontinuités (2002 [1938], 1967 [1934] cf. aussi Leszczyński 2002). Il suppose que le développement du discours scientifique a lieu au moment où l'on commence à penser au sujet d'une réflexion préalablement formulée. Bien sûr, ce processus est exposé à des éclatements et à des ruptures. Elles ne sont pas complètes, dans leur structure, on peut également trouver des discontinuités renouvelées de temps en temps, notamment marquées par un changement de paradigme. Ces discontinuités sont cruciales aussi pour le phénomène de la longue durée (p.ex. la discipline comprise en tant que réflexion sur la réflexion). En effet, elles constituent la base ou les références de la reconstruction. Cela se fait en contradiction avec ce qui est hic et nunc, mais éclairé par « ce qui était », " comment c'était » ainsi qu'avec ce qui pourrait être important pour la construction nouvelle.

La position de Bachelard, qui peut constituer la base de la formulation de lignes directes épistémologiques sur la façon d'utiliser le passé, se fonde sur la perspective de la psychologie de la science. Cette perspective justifie la position selon laquelle « le passé intellectuel doit être connu comme tel, comme passé affectif. Mais finalement pour avoir une conscience de la construction phénoménologique, ce qui est passé devrait être pensé en fonction de ce qui est nouveau » (Bachelard 2002 [1938] : 323) ${ }^{3}$. L'œuvre principale de Bachelard qui peut bien servir à la pensée transformation est l'analyse de ce processus déchiré par des ruptures mais aussi ayant des ressources et une durée dans des discontinuités. La catégorie de la durée est également évidente dans le développement de la science qui, comme dit Bachelard : « n'existe que comme École perpétuelle » (ibidem : $325)^{4}$.

Bref, les prémisses du cadre méthodologique du processus transversal de transformation des activités mentales peuvent également constituer le point de départ pour la construction d'un outil d'analyse de longue durée. En cas de

\footnotetext{
3 Ce mécanisme de constituer une unité dans la diversité est cohérent avec le mécanisme de transformation transculturelle discuté par Welsch (1998a). L'auteur écrit ainsi : « La diversité traditionnelle, contenue ou forme de cultures individuelles, est en effet en train de disparaître de plus en plus. Mais à sa place, un nouveau type de diversité émerge : les cutures et les formes de vie différentes, dont chacune résulte d'une interpénétration transculturelle et a un style transculturel » (ibidem : 217).

4 « Dans l'oeuvre de la science seulement on peut aimer ce qu'on détruit, on peut continuer le passé en le niant, on peut vénérer son maître en le contredisant. Alors oui, l'École continue tout le long d'une vie. Une culture bloquée sur un temps scolaire est la négation même de la culture scientifique. Il n'y a de science que par une École permanente. C'est cette école que la science doit fonder. Alors les intérêts sociaux seront définitivement inversés : la Société sera faite pour l'École et non pas l'École pour la Société » (Bachelard 1967 [1934] : 284).
} 
l'école de pensée, nous posons des questions importantes sur le phénomène de la durée, de sa complexité/dualité, du processus de transmission des normes et des valeurs, de sa constitution comme institution symbolique et du processus de transformation silencieuse. Il est particulièrement important de ne pas négliger l'analyse multidimensionnelle, couvrant la dimension du temps et de l'espace dans des structures complexes.

\section{Durée et transformation}

Aussi bien la durée que la transformation sont importants dans l'œuvre de Bachelard. La durée est liée aux différentes catégories de temps dans lesquelles la science se forme : le temps chronologique avec lequel il est habituel de mesurer et structurer les acquis dans le développement de la science, et le temps logique auquel Bachelard se réfère.

Si nous plaçons ces théories, consécutives, concernant un même phénomène, dans le temps logique, et essayons, même en négligeant leur ordre chronologique, de comparer les définitions de leurs concepts sousjacents/de base, puis de dériver l'un de l'autre, alors il s'avère qu'il y a des ruptures entre certains d'entre eux, c'est-à-dire des moments où apparaît quelque chose qui n'était pas là à l'origine et qui ne peut pas être dérivé du précédent, tout en conservant les mêmes définitions des concepts de base (Leszczyński 2002 : 368-369) ${ }^{5}$.

Si une rupture, pour reprendre le langage de François Jullien $(2009,2017)$, est une modification visible des processus qui se déroulent silencieusement et invisiblement, et qui peuvent parfois être perçus en radicale discontinuité, inclut ces éléments synthétisés qui persistent et se transforment, dans ce cas-là, il n'y a pas de rupture brutale, les deux processus, la durée et la transformation, se déroulent en parallèle. Le processus de durée fait référence aux principaux indices qui identifient une école de pensée donnée, son lexique, ses concepts de base, son point de vue etc. Le processus de transformation consiste à produire des significations et des sens, en fonction de nombreux éléments externes (les plus souvent paradigmatiques, mais aussi contextuels) et internes (liés à la personne du chercheur, à son expression identitaire), que Bachelard souligne si clairement.

Toutefois, ce phénomène de transformation est paradoxal car la rupture de pensée n'arrive jamais complètement, du moins dans un sens symbolique. C'est le cas de la durée de l'école de pensée en pédagogie sociale (Marynowicz-Hetka 2019, 2020).

\footnotetext{
5 C'est une interprétation de la position de Barchelard par le traducteur de son œuvre en polonais Damian Leszczyński.
} 
Une telle interprétation des idées de Bachelard peut être appliquée à une analyse approfondie des processus de construction d'une école de pensée, qui ne se limitent pas à nier les acquis/l'héritage du passé, mais constituent une impulsion importante pour son enrichissement. Cet enrichissement ne consiste pas simplement à " coller » ensemble et à donner de nouvelles significations et de nouveaux sens. Dans ce processus, une fonction particulière est jouée par le point de vue du chercheur. D'un côté, il 'part' des limites, et de l'autre, il en tire une impulsion pour de nouvelles recherches.

\section{Complexité, dualité, liens conjoints ${ }^{6}$}

Le cadre épistémologique de l'approche transversale du processus de transformation des activités mentales comprend les concepts de complexité, de dualité et de liens conjoints formés en spirale, que nous essayons d'inclure dans l'intelligibilité de l'activité dans le domaine de la pratique.

La caractéristique dominante du cadre épistémologique du processus de transformation transversale des activités mentales est sans aucun doute la complexité, dualité structurelle de ce phénomène. D'une part, c'est une certaine manière de percevoir le réel, caractérisée par la saturation des situations sociales de « signes de complexité, issus de l'interpénétration d'influences différentes, de la collision de ce qui passe, et partant avec ce qui annonce son arrivée, qui marque même une nouvelle ère, malgré le fait que l'absence d'une forme prête pour elle est la maturité de ses manifestations » (Witkowski 2013 : 99).

D’autre part, ce cadre épistémologique signale la nécessité de sensibiliser aux liens complexes, non évidents, et conjoints des activités dans le domaine de la pratique. Dans ce contexte, la préparation ou la formation à une telle perception de l'espace de l'activité est également dotée de références culturelles et de son «propre » savoir d'un sujet agissant, pour interpréter la complexité des situations sociales ainsi que les mécanismes de transformation qui s'opèrent en spirale et silencieusement dans le domaine de la pratique (Jullien 2009, 2017).

\section{Traits caractéristiques}

L'approche transversale de l'activité dans le domaine de la pratique se caractérise par plusieurs syndromes, ensembles de caractéristiques.

Le premier est l'interpénétration des perspectives participatives et compréhensives qui en effet donne lieu à l'intelligibilité du sens et de la signification des

\footnotetext{
${ }^{6}$ Le concept élaboré par Jean-Marie Barbier à l'occasion des travaux des recherches qui se sont intéressés « aux corrélations de transformations » (Barbier $2018: 20$ ).
} 
liens conjoints de l'activité humaine des sujets agissant, située dans un contexte désordonné, parfois chaotique, en constante évolution et en cours de construction. Dans cette perspective, le sujet agissant est situé en interne ; en participant dans le champ de pratique qu'il essaie de comprendre. Cette perspective est susceptible d'analyser le réel dans une vue tridimensionnelle, dans laquelle les éléments externes et internes se croisent et s'éloignent du champ de la pratique.

Le deuxième ensemble/syndrome de traits caractéristiques comprend la possibilité d'analyser l'intrication du réel du point de vue de différentes disciplines, mais son message est universel/commun. De cette manière, l'approche discutée combine ce qui est universel/commun et reproductible avec ce qui est individuel, spécifique et exceptionnel. En même temps, son aspect métathéorique et métaméthodologique peut s'avérer utile pour la constitution des cadres d'une discipline et notamment pour le processus de disciplinarisation d'une pratique réflexive, ce qui est le cas de la pédagogie sociale. Cette circonstance est devenue un énorme coup de pouce pour revenir à la réflexion sur l'utilité de l'approche transversale pour analyser le champ d'activité et s'y préparer afin de penser selon le paradigme de transformation transversale. Ceci a paru utile pour une relecture des idées de pédagogie sociale de Radlińska (cf. Marynowicz-Hetka, Laot 2018) synthétisées dans la phrase bien connue : «le nom de pédagogie sociale exprime son rôle du lien entre l'ensemble des sciences de l'éducation et les sciences de la culture et de la société » (Theiss 1984 : 233).

Le troisième syndrome de trait caractéristique de l'approche transversale se réfère à l'accent mis sur le fait que cette approche est susceptible d'analyser le réel social de manière multiréférentielle. Cette approche transversale peut être présente dans les trois dimensions évoquées ci-dessus. Elle consiste en une approche méthodologique particulière d'analyse de pratique et de justification d'une méthode pour construire une métaréflexion sur le champ d'activité. Elle utilise son propre langage métathéorique, dans lequel elle exprime des liens entre les éléments analysés. Elle est susceptible de faciliter le regard complexe qui constitue le second niveau d'analyse après la séparation des facteurs, indispensable au début du processus de connaissance d’un phénomène.

\section{Analyse transversale des activités}

L'analyse transversale de l'activité7, connue dans la littérature sous le terme « entrée activité » (Barbier, Durand 2003) exprime un changement paradigmatique ayant pour but de « proposer pour des termes habituels/courants des formulations

\footnotetext{
7 Le terme " l'analyse transversale des activités » a été proposé par interprète de lédition polonaise du Vocabulaire d'analyse des activités, car il reflète le mieux le sens de l'approche à l'activité qui y est présentée. Cette section d'article est une traduction française modifiée de la postface de ce Vocabulaire... (cf. Marynowicz-Hetka 2016).
} 
à l'intention scientifique ». Cette transformation paradigmatique " rompt » avec l'orientation scientiste et élimine la frontière entre la théorie et la pratique. Les recherches sont perçues en tant qu'accompagnement de l'activité, où le sujet modifie ses fonctions par rapport à l'activité. C'est justement « la découverte de l'activité » qui a montré aux sujets, et plus particulièrement aux chercheurs, les difficultés épistémologiques, qui se résument dans la question : comment faut-il analyser l'activité en prenant en considération un tel réseau d'articulations ?

Les prémisses principales de ce concept d'analyse transversale des activités ont été formulées par Jean-Marie Barbier dans Vocabulaire d'analyse des activités (Barbier 2016, 2017). Elles couvrent de nombreux aspects discutés ci-dessous. Parmi ceux-ci les plus significatifs sont la complexité d'analyse, la dynamique et la processualité de l'activité, de l'activité située en contexte, la construction parallèle de l'activité et d'un sujet.

La nature holistique de l'approche s'exprime par une supposition que les actes singuliers d'action, de perception, de connaissance, d'expression des émotions et de construction de soi, sont inséparables (Barbier 2017 : 43-44). En effet, cette position épistémologique sensibilise à l'analyse de l'activité non seulement du point de vue de ce qui est visible, mais aussi de ce qui directement est invisible, pourtant très important pour le déroulement de l'ensemble du processus d'activité.

La dynamique et la processualité de l'approche de l'analyse transversale de l'activité soulignent une dimension transformationnelle et évolutive des actions, et montrent leur image fonctionnelle. Une particularité de ce concept est l'effort de construire une configuration des actes d'action, et pas simplement de séparer ses champs singuliers. Cela se traduit par la reconnaissance et l'admission de la perspective transversale. Elle donne lieu à l'élaboration des outils d'analyse de l'activité, utiles et possibles à appliquer dans divers domaines et espaces.

En fait, situer l'activité dans un contexte changeant signifie aussi accepter sa variabilité, son évolution et sa transformation. La mise en contexte de l'activité nécessite également de prendre en compte les circonstances externes de sa survenance. La question qui se pose est de savoir : comment lier les dimensions transversale et holistique de cette approche avec sa dimension singulière. L'une des réponses se trouve dans la perception de l'action comme une " organisation singulière d'activités » (Barbier 2017 : 40). Cette catégorie est susceptible à favoriser le travail analytique de recherche, et notamment la découverte de déplacement de significations des activités des participants dans l'interaction.

La construction parallèle de l'activité et du sujet, est une condition très importante non seulement pour créer des savoirs sur l'activité au cours de la recherche entreprise, mais aussi au cours de la construction d'une pratique réflexive par les sujets qui y participent. Cette prémisse justifie d'autres catégories conceptuelles et les significations qui leur sont attribuées. Elles mettent l'accent sur la convergence, l'interdépendance, la réciprocité et l'articulation, grâce auxquelles elles permettent de décrire la richesse et la complexité de l'activité se déroulant 
dans divers champs de pratiques et dans divers espaces. Cette prémisse de construction parallèle de l'activité et construction du sujet, justifie également le but de l'effort entrepris par l'auteur (Barbier 2016, 2017) qui se concentre sur l'attribution des nouvelles significations aux concepts ordinaires. Ce déplacement est extrêmement important car il indique également l'ouverture épistémologique de ce concept.

Dans l'analyse transversale des activités, on distingue deux dimensions d'analyse et les catégories analytiques qui y sont regroupées (Barbier 2017 : 133 138). Ce sont :

- les concepts mobilisateurs. Ils permettent de décrire ce que font les entités dans un espace donné. Ils incluent le langage de l'action, défini comme « lexique de l'action » (ibidem: 133-135). Ce sont les concepts qui déclenchent la prise d'activité. Il est important de reconnaître la complexité de ce type de lexique, qui peut être exprimé dans trois registres de significations : mentale, affective et cognitive ;

- les concepts d'intelligibilité. Ils participent à la construction des savoirs sur les activités de l'intelligibilité des actions. Ils sont définis comme "lexique de l'intelligibilité des actions » (ibidem : 135-138). Ces concepts permettent d'établir des relations entre de nombreux éléments existants, ou plutôt entre leurs représentations. « ...»

En formulant, de manière synthétique, mais aussi sélective, les éléments les plus importants de l'analyse transversale de l'activité, nous soulignons ses dimensions heuristique, holistique et processuelle. Cette approche interpénètre transversalement le concept de transformation. La réflexion sur le processus de transformation, ou «penser les transformations » comme dit l'auteur (ibidem : 194-196), se déroule dans différents espaces. Ce sont : l'espace mental, que nous déclenchons lorsque nous pensons au processus des activités ; l'espace imaginaire lorsque nous réfléchissons et l'espace discursif lorsque nous communiquons le fait d'entreprendre des activités. L'analyse de l'activité couvre de nombreuses relations connexes et conjointes entre ces espaces. Ainsi son résultat sous forme d'énoncés sur des « régularités observables » constitue les savoirs sur l'activité.

L'utilité de la conception transversale d'analyse de l'activité est présente dans la vision socio-pédagogique du champ de la pratique. Elle est notamment présente dans les recherches qui prennent pour objet ce qui pousse un sujet à agir et aussi à analyser les processus de transformations dans le champ de pratique. Cette conception transversale de l'analyse de l'activité permet de découvrir la qualité de ces processus et notamment sa dimension axiologique. Les premières références à ce concept ont trouvé leur place importante dans un manuel de pédagogie sociale (Marynowicz-Hetka 2006), notamment dans la conceptualisation du concept d'action qui devient action sociétale. Différentes activités communes avec les chercheurs de l'équipe de Jean-Marie Barbier ont permis d'approfondir ce sujet et d'élaborer les prémisses du paradigme de la transformation transversale en pédagogie sociale (cf. Marynowicz-Hetka 2020). 


\section{Paradigme de la transformation transversale en pédagogie sociale}

L'ensemble des notions et des concepts, structuré et partagé par les participants d'un champ de pratique qui crée l'approche consciente, peut être appelé 'un paradigme'. Nous n'utilisons ce terme que dans ce sens-là. Cela signifie aussi que nous pensons alors à la pédagogie sociale représentant une réflexion sur la réflexion. Nous nous référons à son discours actuel par rapport à ses premières formulations et au discours contemporain en sciences humaines et sociales.

À la suite de l'analyse ainsi menée, il est possible de définir le paradigme de la pédagogie sociale de manière transversale, c'est-à-dire combinant dans l'unité, et résultant de la diversité, les éléments appartenant à de nombreuses approches. La pédagogie sociale est donc comprise en tant que réflexion sur l'activité dans le champ de pratiques, exprimant de façon complète un certain système de concepts et des significations pouvant constituer une référence pour comprendre cette activité, son orientation, et les raisons de son entreprise.

Cette position permet de maintenir les liens avec les approches des sciences humaines et sociales en mettant en place un point de vue socio-pédagogique, défini et cohérent, conduit transversalement. Parmi les concepts utiles pour construire le paradigme transversal de pédagogie sociale il faudrait en distinguer plusieurs autres discutés dans d'autres textes (cf. Marynowicz-Hetka 2019, 2020). Ce sont notamment : le concept transversal de l'analyse du champ de pratique (Barbier 2016, 2017, 2020) ; des mécanismes pour créer une expérience partagée par l'intégration et la reconstruction de l'expérience individuelle (Dewey 1968 [1947]), qui permet la création d'une institution symbolique partagée par les participants dans le domaine de la pratique ; reconnaissant que l'activité c'est la vie (Barbier 2016, 2017), donc tout ce que nous faisons, en agissant, en changeant l'environnement, en influençant les autres, et en nous changeons nous mêmes (Barbier 2018a).

Toutes les activités et les transformations qui se produisent sont liées dans une boucle en spirale, une tresse (Barbier 2016, 2017). Il n'est donc pas nécessaire de lister des actes unitaires, car la conception transversale est susceptible de favoriser la perception du champ de pratique de manière holistique, complète et complexe, sans oublier la dualité et les oscillations dans le développement processuel des événements.

Les transformations de l'objet de pédagogie sociale, les méthodes d'analyse des phénomènes de compréhension de l'appareil conceptuel, la recherche des raisons de l'orientation de l'action dans le champ de pratique, tout cela subit des modifications vers un vécu intégré et partagé du champ de pratique. Au terme de cette analyse, il convient de souligner l'utilité de mettre l'accent sur les façons d'une telle démarche, tout en gardant à l'esprit les doutes que cette direction de développement de la réflexion sur la réflexion peut apporter. L'estimation positive concerne principalement : 
- une nouvelle lecture de la perspective traditionnelle du positionnement transversal de la pédagogie sociale « entre-deux » autres disciplines humaines et sociales. Cette nouvelle lecture lui permet de prendre une nouvelle dimension, notamment au vu de l'élargissement de son objet et de son hétérogénéité de plus en plus accentuée. Il s'agît vraisemblablement d'un processus inévitable et résultant de l'injonction de mener la recherche en équipes transdisciplinaires et transculturelles utilisant diverses positions épistémologiques. Ce processus tend probablement vers un flou d'indétermination. En effet, trouver l'approche convenable devient le défi significatif pour stabiliser la posture du chercheur, sa conscience de son identité épistémologique ;

- une perception expressive des tendances et des syndromes de traits caractéristiques dans la mise en forme des manières d'analyse des phénomènes, donc aussi des activités liées à la conception d'outils d'analyse et de propositions d'agir. Il s'agit de passer du faire « pour » le sujet, d'évaluer et planifier de l'extérieur, de faire ensemble, coopérer, partager, et enfin de créer une communauté. La dimension de la coopération interactive, du partage des règles et des valeurs pour trouver une manière convenable d'analyser un phénomène, favorise par conséquent la création d'une culture de la pratique dans les espaces de recherche, formation, soins, travail social, donc, dans l'action professionnelle ;

- la clarté d'analyse et du point de vue. Cela signifie, par exemple : qu'on ne parle pas de l'action globalement, mais plutôt de sa dimension socio-pédagogique ; les problèmes sociaux sont analysés moins en termes de description des causes de leur émergence dans une perspective macrostructurale, mais davantage en tant que réflexion sur les solutions possibles pour apercevoir et anticiper la menace au développement individuel et collectif. Des outils sont également construits, ils permettent d'analyser des activités et d'imaginer le déroulement du processus d'insertion sociale et de croissance de l'individu dans son milieu de vie ;

- une conviction que parmi les variants possibles d'orientation des démarches de recherche, particulièrement utiles peuvent être ceux qui permettent une compréhension processuelle du réel et donne lieu à co-créer des projets de transformation.

\section{Boucle de relations : entre expérience partagée et pensée}

Construire une pensée de transformation est constamment évolutif. Elle se caractérise par des ruptures paradigmatiques, mais aussi personnelles, institutionnelles et des discontinuités, grâce auxquelles une idée persiste malgré les obstacles.

Nous nous intéressons à la manière dont ce phénomène relationnel d'expérience partagée (Dewey 1968 [1947]), est créé, que les participants à l'activité ressentent comme une posture de pensée commune et propre à eux- 
mêmes. Il convient d'étudier ce processus qui s'exprime en partage d'expériences et l'élargissement de son champ d'application en fonction des attentes individuelles et des circonstances du contexte social.

Ce phénomène relationnel d'expérience partagée est exposé à des ruptures et des discontinuités, dont les traits ont souvent une dimension paradoxale. Grâce à ces processus, la posture de pensée subit des transformations et des changements, et par conséquent elle n'est ni monumentale ni ossifiée. L'acceptation de cette transformation perpétuelle, multi-complexe et liée est pour ses participants une caractéristique importante qui les protège devant d'éventuelles crises et ruptures. Ils sont susceptibles de construire du nouveau, grâce à des processus de discontinuité, et ils permettent de découvrir des prémisses de base qui identifient cette posture de pensée.

Les travaux de recherche sur la question de " penser transformation » nous ont sensibilisé à un trait très important pour la perspective socio-pédagogique, notamment celui de souligner la processualité de transition, de transformation. Dans cette perspective, il est extrêmement important de penser à l'activité dans le champ de pratiques en fonction de ce qui se passe et comment cela se passe. C'est une question qui tient une place importante dans la pédagogie sociale, mais c'est un sujet pour un autre article.

\section{Bibliographie}

Bachelard G. (2002 [1938]) Kształtowanie umysłu naukowego. Przyczynek do psychoanalizy wiedzy obiektywnej, trad. D. Leszczyński, Gdańsk, Słowo/obraz terytoria.

Barbier J.-M. (2006) Działanie w kształceniu i pracy socjalnej. Analiza podejść, trad. G. Karbowska, Katowice, Wydawnictwo Naukowe „Śląsk”, coll. BPS.

Barbier J.-M. (2016) Leksykon analizy aktywności. Konceptualizacje zwyczajowych pojęć, trad. et élaboration E. Marynowicz-Hetka, Łódź, Wydawnictwo Uniwersytetu Łódzkiego.

Barbier J.-M. (2017) Vocabulaire d'analyse de l'activité, Penser les conceptualisations ordinaires, 2e édition revue et augmentée, Paris, Presses Universitaire de France.

Barbier J.-M. (2018) Penser en termes de transformation in : J.-M. Barbier, M. Durand (coord.), avec la collaboration de C. Cohen, M.-L. Vitali, Représenter/transformer. Débats en analyse des activités, Paris, L'Harmattan : 11-23.

Barbier J.-M. (2020) La transformation continue par les sujet des rapports qu'ils établissent avec/entre les composantes de leur activité in : Comprendre/transformer Débats en éducation et Formation, J. Thievenaz, J.-M. Barbier, F. Saussez (dir.), Bern, Berlin, Bruxelles, New York, Oxford, Warszawa, Peter Lang : 9-17. 
Barbier J.-M., Durand M. (2003) L'activité : un objet intégrateur pour les sciences sociales? «Recherche \& Formation », $\mathrm{n}^{\circ} 42$, L'analyse de l'activité. Approches situées, J.-M. Barbier, M. Durand (dir.) : 99-117, https://doi.org/10.3406/refor.2003.1831

Barbier J.-M., Galatanu 0. (2006) Jednostkowość działania: kilka narzędzi analizy in : J.-M. Barbier, Działanie w kształceniu i pracy socjalnej. Analiza podejść, trad. G. Karbowska, Katowice, Wydawnictwo Naukowe „Śląsk”, coll. BPS : 145-174.

Braudel F. (1949) La Méditerranée et le monde méditerranéen à l'époque de Philippe II, Paris, Armand Colin.

Braudel F. (1997 [1958]) Les Écrits de Fernand Braudel, vol. 2 : Les Ambitions de l'histoire, Paris, De Fallois.

Braudel F. (1999 [1971]) Historia i trwanie, trad. B. Geremek, W. Kula, Warszawa, Czytelnik.

Dewey J. (1968 [1947]) Expérience et éducation, trad. M. A. Carroi, Paris, Librairie Armand Colin.

Foucart J. (2016) Fluidité sociale et conceptualisations de l'entre-deux. Systèmes semichaotiques, réseaux et transactions sociales, Essai, Aix en Provence, Les éditions Persée.

Jullien F. (2009) Les transformations silencieuses, Paris, Éditions Grasset et Fasquelle.

Jullien F. (2017) Une seconde vie. Commencer véritablement d'exister, Paris, Éditions Grasset.

Leszczyński D. (2002) Filozofia nauki Gastona Bachelarda in : G. Bachelard, Kształtowanie umysłu naukowego, trad. D. Leszczyński, Gdańsk, Słowo/obraz terytoria : 328-377.

Kubicki R. (1998) O skoku do Królestwa Rozumu Transwersalnego in : Filozoficzne konteksty rozumu transwersalnego. Wokół koncepcji Wolfganga Welscha, deuxième partie, R. Kubicki (dir.), Poznań, Wydawnictwo Fundacji Humaniora : 161-168.

Marynowicz-Hetka E. (2006) Pedagogika społeczna. Podręcznik akademicki. Wykład, vol. 1, Warszawa, Wydawnictwo Naukowe PWN.

Marynowicz-Hetka E. (2008) Towards the transversalism of social pedagogy, trans. B. Przybylska in : Reframing the Social. Social Work and Social Policy in Europe/ Reframing des Sozialen. Soziale Arbeit und Sozialpolitik in Europe/Reframing del Sociale. Lavoro Sociale e Politica Sociale nell'Europa, F. W. Seibel, H.-U. Otto, G. F. Friesenhahn (Hrsg.), Boskovice 2008, Albert : 85-102.

Marynowicz-Hetka E. (2016) Konceptualizacje zwyczajowych ram myślenia o aktywności-na marginesie pracy nad tekstem in : J.-M. Barbier, Leksykon analizy aktywności. 
Konceptualizacje zwyczajowych pojęć, trad. et élaboration E. Marynowicz-Hetka, Wydawnictwo Uniwersytetu Łódzkiego : 271-289.

Marynowicz-Hetka E. (2019) Pedagogika społeczna. Pojmowanie aktywności w polu praktyki, Łódź 2019, Wydawnictwo Uniwersytetu Łódzkiego.

Marynowicz-Hetka E. (2020) Social Pedagogy. Comprehending activity in the field practice, trad. M. Hinton, Beau Bassin, Lambert Academic Publising.

Marynowicz-Hetka E., Laot F. F. (2018) Éducation des adultes et progrès social selon Helena Radlińska in : Pionnières de l'éducation des adultes. Perspectives internationales, F. F. Laot, C. Solar (dir.), Paris, L'Harmattan : 163-182.

Radlińska H. (1935) Stosunek wychowawcy do środowiska społecznego. Szkice z pedagogiki społecznej, Warszawa, Nasza Księgarnia.

Skarga B. (2009) Tercet metafizyczny, Kraków, Wydawnictwo Znak.

Theiss W. (1984) Helena Radlińska, Warszawa, Wydawnictwo „Żak”.

Welsch W. (1998a) Transkulturowość. Nowa koncepcja kultury in : Filozoficzne konteksty koncepcji rozumu transwersalnego. Wokół koncepcji Wolfganga Welscha, trad. B. Susła, J. Wietecki, R. Kubicki (dir.), Poznań, Wydawnictwo Fundacji Humaniora : 195-222.

Welsch W. (1998b) Wielość a niezróżnicowanie in : Filozoficzne konteksty koncepcji rozumu transwersalnego. Wokół koncepcji Wolgana Welscha, trad. B. Susła, J. Wietecki, R. Kubicki (dir.), Poznań, Wydawnictwo Fundacji Humaniora : 97-100.

Welsch W. (2015) L'esthétique de Schiller reconsidérée. "La Beauté est liberté dans le phénomène " ou l'esthétique comme enjeu pour pensée moderne, trad. L. Marcucci, « Nouvelle revue d'esthétique », n 15 : 83-92, https://doi.org/10.3917/nre.015.0083

Witkowski L. (2013) Przełom dwoistości w pedagogice polskiej. Historia, teoria, kryty$k a$, Kraków, Oficyna Wydawnicza „Impuls”.

Witkowski L. (2014) Niewidzialne środowisko. Pedagogika kompletna Heleny Radlińskiej jako krytyczna ekologia idei, umysłu i wychowania. O miejscu pedagogiki w przełomie dwoistości w humanistyce, Kraków, Oficyna Wydawnicza „Impuls”.

\section{Sources d'Internet}

Bachelard G. (1967 [1934]) La formation de l'esprit scientifique. Contribution à une psychanalyse de la connaissance objective, 5e édition, Paris, Librairie philosophique J. VRIN, Coll. Bibliothèque des textes philosophiques, http://dx.doi.org/doi:10.1522/ 030331552 [consulté : 19.01.2021]. 
Barbier J.-M. (2018a) Expérience, transformation de soi, construction du moi, affirmation du je, "The Conversation FR", http://theconversation.com/experiencetransformation-de-soi-construction-du-moi-affirmation-du-je-88935 [consulté : 10.01.2018].

Barbier J.-M., Dutoit M. (2021) Apprendre : Etre plus grand dans sa tête, https:// www.innovation-pedagogique.fr/article8610.html [consulté : 13.01.2021].

\section{Transversal Activity Analysis and the Transformation Paradigm}

\section{Summary}

The content of this article shows how the transversal approach to the analysis of activity and the paradigm of transformation, present in the work of Jean-Marie Barbier, permeate the socio-pedagogical perspective. The article also reveals transformations of mental activities in the face of new concepts. The text discusses in turn:

- the epistemological and methodological framework of the transversal approach and its relations with the transformation paradigm;

- the concept of the transversal analysis of activity and its relationship with the transversal paradigm of transformation in social pedagogy.

With regard to Wolfgang Welsch's key concept of "transversal reason", the category of transversality is analysed. The significant features of transversal reason are emphasized: tensions and transformations, which make its image hybrid and paradoxical: causing tensions, but also existing thanks to them. As a result, another category is introduced in the text: "the process of transculturality", which supports the construction of the transversal dimension and learning about the mechanisms of transformation. For this reason, this category seems attractive for exploring the complexity of the "thinking transformations" (pensée transformation) paradigm.

The epistemological and methodological framework of the activity transformation process contains three categories: long duration, rupture, and discontinuity, enriched with relationships of duration and change; complexity, duality and interconnectedness (liens conjoints). Against their background and in relation to them, the concept of the transversal analysis of activity, developed by Jean-Marie Barbier, is discussed. This provides a natural transition to discussing the characteristics of the transversal paradigm of transformation present in social pedagogy.

The conclusion of the text is dominated by the thesis of the evolutionary and changing dimension of thinking about transformations. Paradigmatic breaks, both personal and institutional, are characteristic, but the discontinuity that dominates in every transformation process is a "reservoir of duration", thanks to which ideas exist and can renew themselves. This position at the end of the paper also defines the epistemological dimension of the transversal paradigm of transformation, discussed from a socio-pedagogical point of view. 


\section{Transwersalna analiza aktywności i paradygmat transformacji}

\section{Streszczenie}

Treść tego artykułu pokazuje, w jaki sposób transwersalne podejście do analizy aktywności i paradygmat transformacji, obecne w twórczości Jeana-Marie Barbiera, przenikają perspektywę społeczno-pedagogiczną. Artykuł ujawnia także transformacje aktywności mentalnych w zderzeniu z nowymi konceptami. W tekście omówione są kolejno:

- ramy epistemologiczne i metodologiczne podejścia transwersalnego oraz jego związki z paradygmatem transformacji;

- koncepcja transwersalnej analizy aktywności oraz jej związki z transwersalnym paradygmatem transformacji w pedagogice społecznej.

W odniesieniu do kluczowej koncepcji Wolfganga Welscha, „rozumu transwersalnego", analizowana jest kategoria transwersalności. Podkreśla się znaczące cechy rozumu transwersalnego: napięcia i transformacje, co powoduje, że jego obraz jest hybrydalny i paradoksalny: wywołuje napięcia, ale i istnieje dzięki nim. W rezultacie wprowadza się w tekście kolejną kategorię: „proces transkulturowości”, który sprzyja konstruowaniu wymiaru transwersalnego i poznaniu mechanizmów transformacji. $\mathrm{Z}$ tego powodu ta kategoria wydaje się być atrakcyjna dla odkrycia złożoności paradygmatu : „myśląc transformacje” (pensée transformation).

Ramy epistemologiczne i metodologiczne procesu transformacji aktywności opisują trzy kategorie: „długie trwanie” (longue durée), „zerwanie” (rupture), nieciągłość (discontinuité), wzbogacone o związki trwania i zmiany; złożoności, dwoistości i powiązań wzajemnych (liens conjoints). Na ich tle i w odniesieniu do nich omawiana jest koncepcja transwersalnej analizy aktywności, opracowana przez Jeana-Marie Barbiera. Stanowi to naturalne przejście do omówienia cech charakterystycznych transwersalnego paradygmatu transformacji, obecnego w pedagogice społecznej.

W zakończeniu tekstu dominuje teza o ewolucyjnym i zmiennym wymiarze myślenia o transformacjach. Charakterystyczne są zerwania paradygmatyczne, także personalne i instytucjonalne, jednak dominująca w każdym procesie transformacji nieciągłość (discontinuité) stanowi „rezerwuar trwania”, dzięki któremu idee istnieją i mogą odnawiać się. Stanowisko to zawarte na zakończenie tego artykułu określa też epistemologiczny wymiar transwersalnego paradygmatu transformacji, omawianego ze społeczno-pedagogicznego punktu widzenia.

\section{Pour citer cet article}

Marynowicz-Hetka E. (2021) Analyse transversale des activités et paradigme de la transformation, „Nauki o Wychowaniu. Studia Interdyscyplinarne” 2(13), 48-65, https://doi.org/10.18778/2450-4491.13.05 